\title{
Scoring System Approach for Assessment of Critical Illness using Mobile Phones
}

\author{
Karupothula Madhavi Latha ${ }^{1}$, Shiramshetty Gouthami ${ }^{2}$,P. Ranjith Kumar ${ }^{3}$ \\ Computer Science Engineering, JNTUH, Jayamukhi Institute of Technological \\ Sciences \\ Narsampet, Warangal, Andhrapradesh-506332, India \\ ${ }^{1}$ madhukarupdyahoo.co.in \\ 2 gouthami.shiramshetty@gmail.com \\ 3 ranjith0531@gmail.com
}

\begin{abstract}
This paper demonstrates the use of mobile phones in assessing the illness of patients by developing a Scoring System where medical practitioners (often nurses) collect various physiological signals like ECG, EEG, SpO2, temperature, continuous blood pressure and subjective parameters like level of pain, level of alertness, awareness, behavioral responses etc. After taking the data, a scoring system is utilized for early detection of critical illnesses. The mechanism of scoring is performed either manually, where the medical practitioner ticks on to a scoring board or is automated by relocating the information from scoring board to a PC, where the software performs the scoring calculation. In the proposed system, the medical practitioner inputs the parameters directly on to their mobile phone by collecting the parameters from the patient. The score is automatically calculated by miniature java based software running inside the mobile phone. Based on the score, level of urgency is determined by the intelligent program. At the end, specialists are contacted automatically by messaging services. Moreover, the results of the scoring are transmitted to the hospital server. Therefore, assistance from civilians with mobile phone based medical intelligence can save precious life.
\end{abstract}

\section{Keywords}

- $\quad$ Scoring System, Arterial Blood Gas, Early Warning Score, Glasgow Coma Scale, Hospital Server, Mobile Phone programming, Remote Monitoring, telemonitoring.

\section{Introduction}

Many of the hospitals uses a specific scoring technique, often referred to as Early Warning Scores (EWS) or calling criteria systems for the early detection of critical illness.In this paper, we introduce the concept of mobile phone based EWS, calling criteria and hospital server communication that is suitable for remote monitoring of patients at a war or disaster affected zone. The whole system automates the manual processing of hospital scoring system that usually requires prior experience and expertise of the patient monitoring person. EWS tracks variation of physiology and provides warning for deteriorated physiological balance. An aggregated physiological score or weighted score provides the combined information of blood pressure, pulse, respiratory rate, oxygen saturations, temperature, urine output and level of consciousness. This simplified level of consciousness includes four major factors containing Alert, Respond to voice, Respond to pain, Unconsciousness (AVPU). This scoring system comprising of the flow diagram, the interval for patient review as well as when and where to contact for specialist assistance, is well documented. We design the Scoring System using

DOI : 10.5121/ijdps.2012.3129 
MIDlets. MIDlets can be developed on Java 2 Micro Edition (J2ME) platform following well documented procedures. After the development, MIDlets can be deployed on to the mobile phones supporting Java technologies. Almost all of the recent mobile phones support execution of MIDlets, since these mobile phones are supplied with miniature runtimes called Kilobyte Virtual Machine (KVM) . We have successfully developed MIDlets, to perform health monitoring tasks engaging the processing power of mobile phones, for remote monitoring of patients. Remote monitoring is crucial for war zone or disaster affected area where proper hospital facilities are of scarcity. The Wireless Application Protocol (WAP) is the mechanism used here in case of integration of client (Mobile) and Server (Web Server). Apache Tomcat is the web server used for the interaction with Database Server.

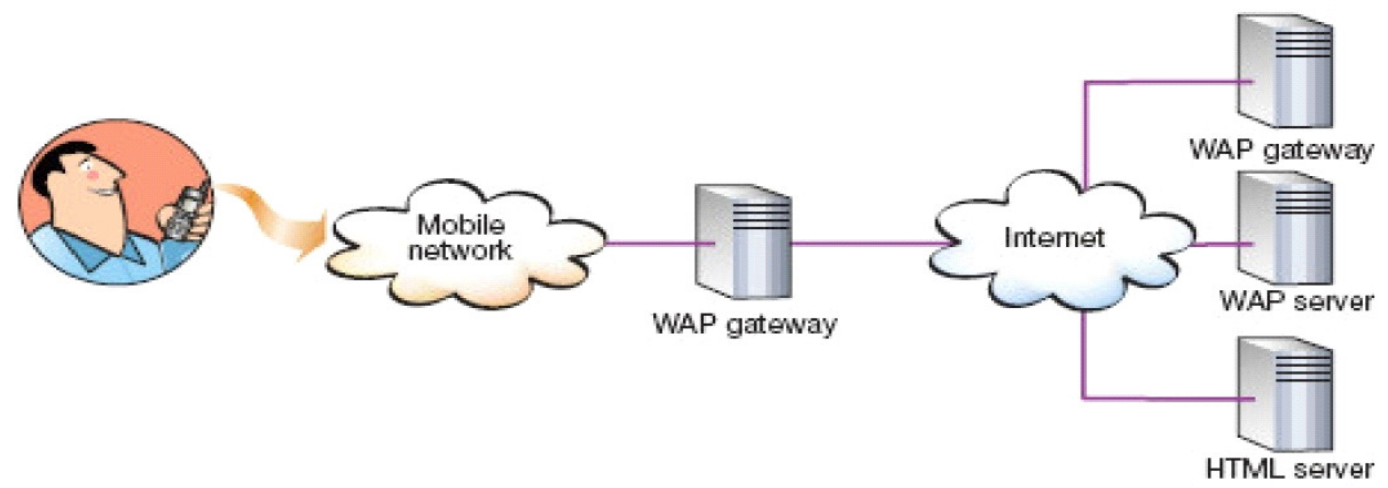

Fig 1 Interface b/w Client and Webserver

\section{Sub Systems:}

The sub-systems under development are

- A Central Information Repository with database of the Patient information and other resources/services

- Web server: A Web server is a program that, using the client/server model and the World Wide Web's Hypertext Transfer Protocol ( HTTP ), serves the files that form Web pages to Web users (whose computers contain HTTP clients that forward their requests) 
- $\quad$ SMS interface for receiving/sending SMS to $2 \mathrm{G}$ Mobile systems, which receives the SMS, converts the SMS into a query and executes the query. The results are then sent as an SMS reply.

- WAP Gateway for linkage with a GPRS/3G mobile. The gateway server translates mobile phone requests (WAP) into HTTP requests and sends them to Web server. The Web server processes the request, and sends WML to gateway server, which in turn sends the WML to phone in the binary compressed WML format

- Localization Module for providing interface for translation.

Amongst the many ICT options available to govt to improve the efficiency\&effectiveness of its delivery process of primary health care, mobile \& wireless technologies offer some exciting opportunities for a low cost, high reach service. There is strong evidence that mobile technologies could be instrumental in addressing slow response rates of govt to citizen requests, poor access to services, particularly for low-income and marginalised populations in underserviced rural areas. In addition, mobile technologies offer significant opportunities for improving the back-office operations of govt. In addition, many primary healthcare clinics located in the rural areas do not have any electronic systems at all \& continue to operate paperbased systems, resulting in patient records being kept by patients themselves. The impact of the use of multiple systems is that it is difficult\& costly to develop a national overview of patient statistics. On a more basic level, it is extremely difficult for idividual institutions within the healthcare sector to share information between each other. One of the clearest examples of this is to be found in the sharing of patient laboratory results. Currently in most instances, this only takes place through manual exchange. Many vendors of Cellular phones started to embed a variety of ehealth services in Mobiles.

\subsection{Modules}

i. APVU / EWS Scoring.

ii. GCS Scoring.

iii. ABG Scoring.

iv. Hospital Server

v. Mobile to Hospital Server Communication.

vi. Mobile Phone Programming Techniques

\subsection{Modules Description:}

\section{i. AVPU / EWS Scoring:}

AVPU scoring system is a MIDlet application which serves an aggregated physiological score or weighted score providing the combined information of systolic blood pressure, heart rate, respiratory rate, body temperature. This simplified level of consciousness includes four major factors containing Alert, Respond to Voice, Respond to Pain, Unconscious (AVPU). When a certain score is attained, expert assistance is sought following a set protocol. This scoring system comprises of the Table, the interval for patient review as well as when and where to contact for specialist assistance, which is well documented. The aim of this project is to evaluate the ability of an EWS to identify medical patients at risk; and to examine the feasibility of EWS as a screening tool to trigger early assessment and admission to an HDU or ICU. 
International Journal of Distributed and Parallel Systems (IJDPS) Vol.3, No.1, January 2012

\section{ii. GCS Scoring:}

The Glasgow Coma Scale or the Glasgow Coma Score is a neurological scale which provides a reliable, objective way of recording the conscious state of a person, for initial as well as continuing assessment. GCS was initially used to assess level of consciousness after head injury and the scale is now used by first aid and doctors as being applicable to all acute medical and trauma patients. In hospital it is also used in chronic patient monitoring, for instance, in intensive care. GCS is ascertained by three types of behavioral responses including eye opening (4 scoring values), verbal response (5 scoring values) and motor response (6 scoring values). GCS values ranges from 3 (deep coma state) to 15 (normal). When GCS values start to decrease from 12, concern is raised. Coma state is confirmed when GCS value is less than 8.

\section{iii. ABG Scoring:}

Arterial blood gas sampling is a medical technique used to check gas levels in the blood. This technique is useful for making sure that certain parts of the blood's chemistry are normal. This technique is commonly used on patients whose breathing is controlled by a mechanical respirator or who are having serious difficulties with breathing. Based on the level of $\mathrm{pH}$, $\mathrm{PaCO} 2$ and $\mathrm{HCO} 3$ in arterial blood, the MIDlet can determine nine different acid-base disorders by scoring (Respiratory acidosis, Metabolic acidosis, Respiratory alkalosis, Metabolic alkalosis, Respiratory acidosis with metabolic compensation, Metabolic acidosis with respiratory compensation, Respiratory alkalosis with metabolic compensation Metabolic alkalosis with respiratory compensation, Mixed metabolic and respiratory alkalosis).

\section{iv. Hospital Server:}

The hospital server supports both message based communication (SMS / MMS) and web server based (HTTP) communication. SMS and MMS communications are possible because a mobile phone is attached to the hospital server via USB (say Nokia N91). Nokia S60, SDK 3rd Edition exposes the required APIs for SMS and MMS communication through the attached phone. The hospital server executes dynamic PHP pages (frontend), which is responsible for the responding of user queries. Different patient reports (in PDF) are generated. These dynamic pages also receive score submission from patient attendees. When the mobile phone submits scores via HTTP, the values are saved within the MySQL database (backend).

\section{v. Mobile to Hospital Server Communication:}

The server is contacted by the mobile phone when calling criteria are encountered. During different stages of scoring, several medical professionals might need to be contacted by the automated scoring mechanism. The server contains the addresses and contacts of the medical personnel. Therefore, the mobile phone only transmits messages to the hospital server that retrieves the contact details of the appropriate personnel to be notified. Each of the messages contain five major parts namely the patient ID, patient attendee ID, scoring type, scoring value and time stamp. Messages are sent via HTTP. The Servlet that handles the MIDlet interaction performs various database based queries. The server creates different patient reports (in PDF) and stores in server. When server receives score submission from patient attendees from the mobile phone, submits scores via HTTP, the links are saved within the MySQL database (back end). This server Communication is used for connecting the server to the mobile client using Stream Socket Connection. For the connection the remote desktop IP address is given in the mobile and then connected. Jar file is created using J2ME wireless toolkit \& installed into the cellular phone through the USB port. 
We can consider three scenarios in this communication.

In the first scenario, the mobile phone transmits the scoring message to the hospital server. Hospital server receives the message and transmits an acknowledgment back to the mobile phone. Since the mobile phone realizes this scenario as a successful message transmission, no further action is performed. In the second scenario, the transmitted message to the hospital server is lost. Therefore the hospital server does not receive anything and does not transmit any acknowledgement message to the mobile. After a timeout period, when the mobile phone does not receive any reply (acknowledgment) from the hospital, it resends the message. The third scenario is similar to the second scenario. Resend action is performed by the mobile, since the reply message from the hospital server (following the receipt of the message from the mobile) is lost.

\section{vi. Mobile Phone Programming:}

NetBeans IDE with NetBeans Mobility Pack can be utilized to program MIDlets for automated calculation of scoring. The scoring can be direct input from the patient attendee or automated input from acquisition device. Some medical acquisition devices support Bluetooth or WiFi connectivity, like Alive Pulse Oximeter or Alive Heart Monitor. Both of them are capable of transmitting the physiological signal directly to regular mobile phones [via MIDlet] or smart phones [via .Net Compact Framework programs]. Implementation of medical scoring is straight forward with Netbeans environment . After development of the MIDlet, it can be successfully debugged and executed on simulated phone environment (in PC). Later on, following the Over the Air (OTA) specification, the MIDlets can be deployed in the mobile phone. J2ME's high level APIs (Form, List, Text Field Item, Choice Group Item, String Item and Date Field Item) were exploited for programming scoring mechanism on mobile phone.

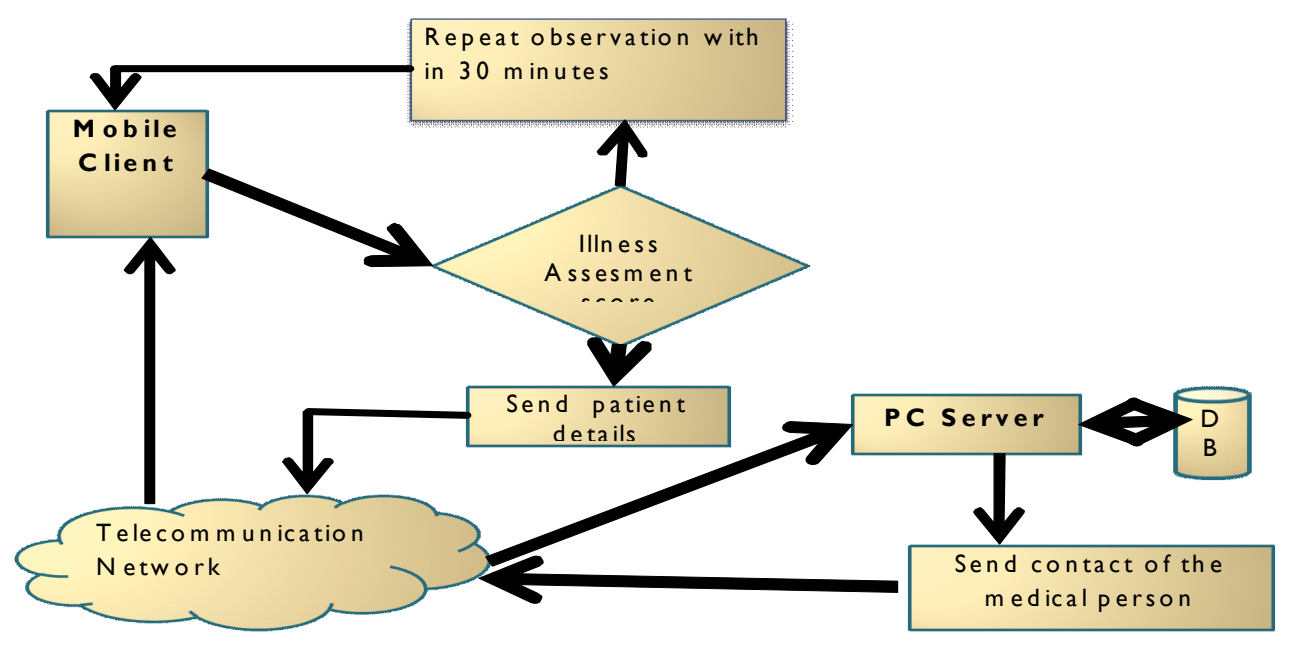

Fig.2 Flow Diagram of mobile phone based scoring mechanism 
International Journal of Distributed and Parallel Systems (IJDPS) Vol.3, No.1, January 2012

\section{Previous work}

Typical group decision support systems (GSS) are implemented in a room that contains a network of computers and public display screens, where multiple participants can meet together to solve problems at the same time. GSS can support multiple participants working on unstructured problems, and facilitate participants to generate ideas, analyze issues and rank alternatives in a group meeting. However, this typical method may be inappropriate for decision makers, with new situations and technologies appearing in recent years. With the changes in the global competitive arena, group decision making needs to become more convenient and efficient in order to complete the task in a timely manner.

\section{Present Work}

Advanced wireless technologies facilitate group decision making conducted from a wired network to a wireless network. The main objective of this paper is to implement Scoring system with wireless technology, where emergency patients can be monitored and treated quickly. In a non hospital scenario, where patients are monitored, attended and treated by amateur volunteers, conformance to EWS / Calling Criteria needs further technological assistance. The Scoring System serves automated calculation of EWS which will benefit the volunteers in camps near a war or disaster zone. The whole system automates the manual processing of hospital scoring system that usually requires prior experience and expertise of the patient monitoring person. With this system in place, the naive user only provides required inputs inside his mobile phone, and the mobile phone automatically decides when and what action to take. In case, of an extreme scoring value, the mobile phone retrieves the contact details of the specialist requiring attention from the hospital server. The particular medical expert is then notified and informed about the deteriorating condition of the patient. 
International Journal of Distributed and Parallel Systems (IJDPS) Vol.3, No.1, January 2012

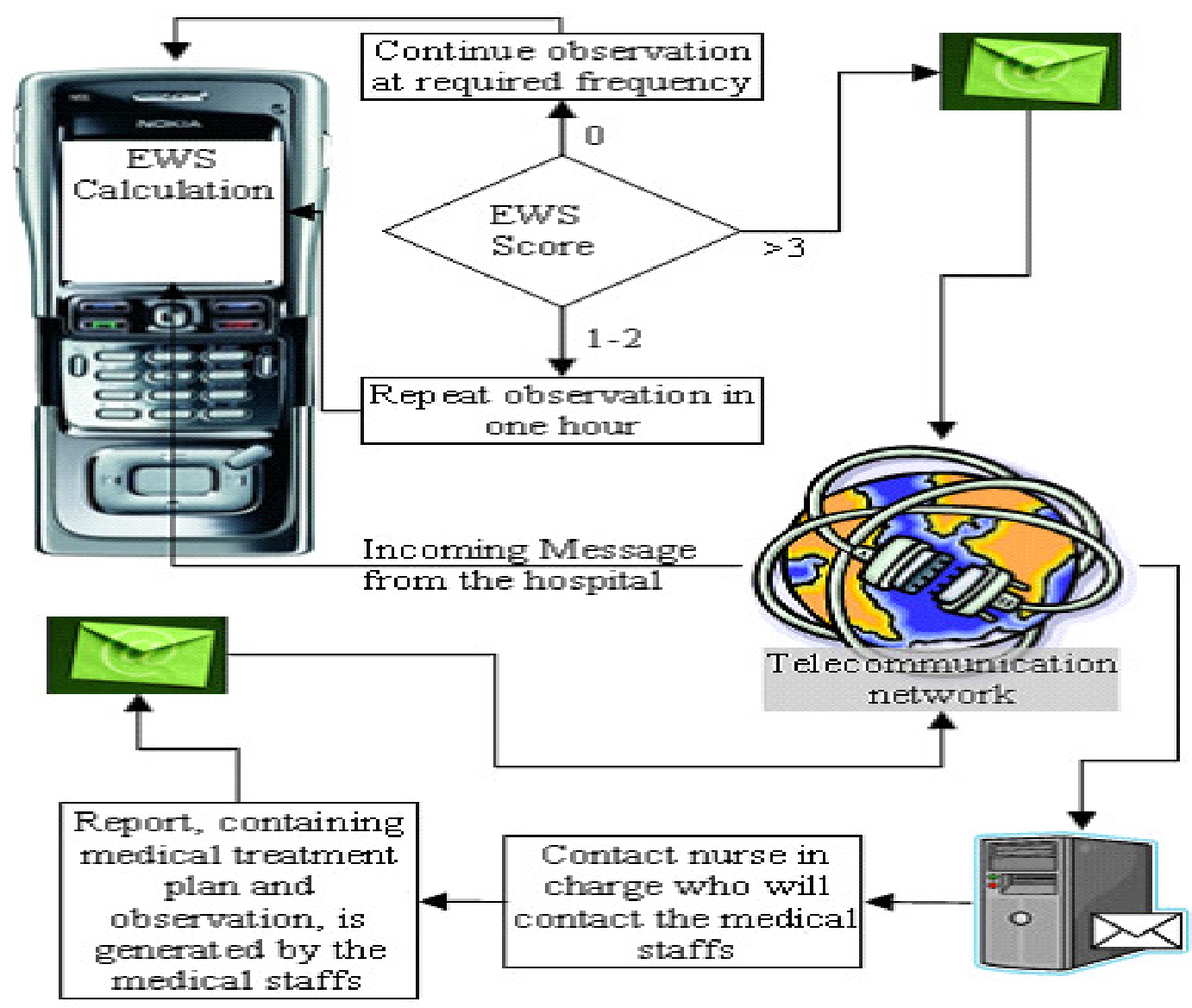

Fig.3 System architecture of mobile phone based scoring mechanism

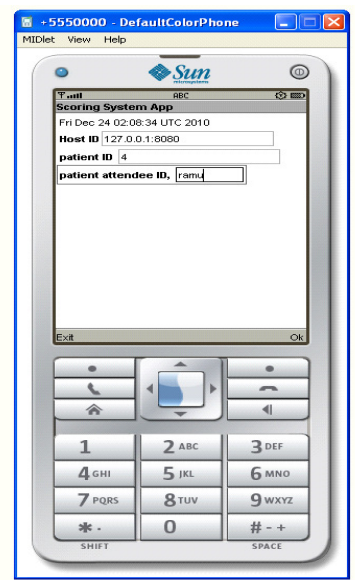

Fig.4 Login to Scoring System 


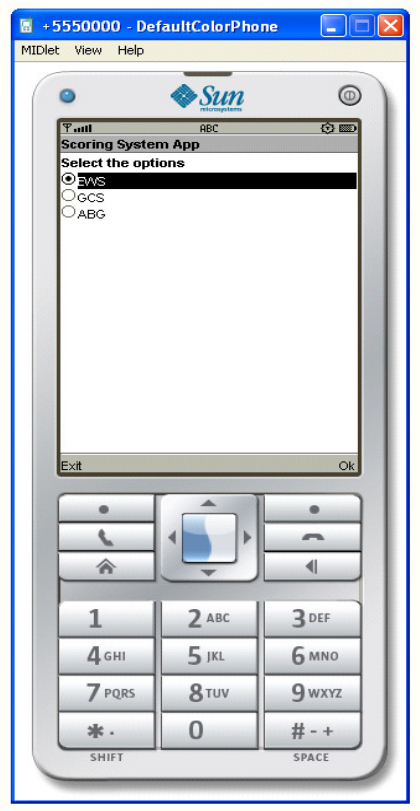

Fig.5 Selecting an Option in Scoring System

\section{Conclusions and Future Work}

We conclude that wireless technology has shifted the doctor centric health care approach to the patient centric healthcare. Before 21 st century, heart rate was calculated by the doctors, wearing stethoscopes. However, in recent days watches that automatically calculate heart rate have evolved. People are using Blood Pressure Machine, Diabetes Checking Machine, Wearable ECG, Pulse Oximetry etc. from their home rather than consulting a doctor. Modern society is more aware and knowledgeable about their health than ever before. The proposed scoring system is another exposure of doctor's knowledge to patient (or patient attendee) by utilizing mobile phone. With the exponential growth of mobile phone usage, total number of mobile phone connections will soon reach the total world population. Transmission of health messages (through SMS, MMS, and HTTP) with medical scoring MIDlets will certainly bring about revolution for remote patient care. We have successfully demonstrated the feasibility of running MIDlets inside the mobile phone for identification of critical illness and possible health breakdown. In many of the cases, inexpensive mobile phones can substitute the requirement of an additional computation platform for medical information processing tasks. Most importantly, people unaware of the medical domain can now step forward for patient monitoring, since medical knowledge can be embedded within the mobile phone.

\section{Acknowledgments}

We extremely thank our Principal and the management for their continuous support in Research and Development. We are also very grateful to our faculty members for their valuable suggestions and their ever ending support. Especially, we thank our college Principal and management for their financial support for receiving the sponsorship. 
International Journal of Distributed and Parallel Systems (IJDPS) Vol.3, No.1, January 2012

\section{References}

[1] Shin-Yuan Hung, Chia-An Tsai, Pei-Jeng Dai,"Development and Applications of Wireless Group Support Systems in MobileBusiness", 2009 Eighth International Conference on Mobil Business

[2] R. Agarwal, E. Karahanna, Time flies when you're having fun: Cognitive absorption and beliefs about information technology usage, MIS Quarterly 24 (4), 2000, pp. 665-694.

[3] J. J. Baroudi, W. J. Orlikowski, A short-form measure of user information satisfaction: A psychometric evaluation and notes on use,Journal of Management Information Systems 4 (4), 1988, pp. 44-59.

[4] I. Cil, O. Alpturk, H. R. Yazgan, A new collaborative system framework based on a multiple perspective approach: Inteliteam, Decision Support Systems 39 (4), 2005, pp. 619-641.

[5] C. W. Holsapple, A. B. Whinston, Decision support systems: A knowledge-based approach, West Publishing Company Minneapolis/St. Paul, 1996.

[6] R. T. Watson, G. DeSanctis, M. S. Poole, Using a gdss to facilitate group consensus: Some intended and unintended consequences, MIS Quarterly 12 (3), 1988, pp. 463-478.

[7] C. P. Subbe, R. G. Davies, E. Williams et al., "Effect of introducing the modified early warning score on clinical outcomes, cardiopulmonary arrests and intensive care utilisation in acute medical admissions",Anaesthesia 58, 2003, 775-803

[8] Resuscitation Council (UK), Advanced Life Support 5th edn., Resuscitation Council UK, 2006, London

[9] P. Jevon and B. Ewens, "Monitoring the critically ill patient", 2nd Ed.,Blackwell Publishing, Oxford, UK, 2007, ISBN: 978-1-4051- 4440-7

[10] NCEPOD , An Acute Problem? National Confidential Enquiry into Patient outcome and Death, 2005, London.

[11] F. Sufi, "Mobile phone programming with Java 2 Micro Edition", Proceedings of the 2007 International Workshop on Mobile ComputingTechnologies for Pervasive Healthcare, Phillip Island, Melbourne,Australia, December, 2007, pp. 64-80, ISBN: 978-0- 646-48230-9

[12] F. Sufi, Q. Fang, S. S. Mahmoud and I. Cosic, "Heartbeat.NET: A realtime health monitoring system”, Big Book of Idea,Commercialisation Expo 2006, Melbourne, Australia, June 2006

[13] F. Sufi, Qiang Fang, Seedahmed S. Mahmoud and Irena Cosic, "A mobile phone based intelligent telemonitoring platform", $3^{\text {rd }}$ IEEEEMBS International Summer School and Symposium on Medical Devices and Biosensors (ISSS-MDBS 2006), Massachusetts Institute of Technology, Cambridge, Boston, MA, USA,September, 2006

[14] F. Sufi, Q. Fang and I. Cosic, "ECG R-R peak detection on mobile phones", 29th Annual International Conference of the IEEE Engineering in Medicine and Biology Society, Lyon, France, August, 2007

[15] C. Gwinnutt, Clinical Anaesthesia 2nd edn. Oxford: Blackwell Publishing, 2006

[16] D. Fairley, "Using a coma scale to assess patient consciousness levels",Nursing Times 101 (25), 2005, 38-47

[17] K. Allen, "Four step method of interpreting arterial blood gas analysis", Nursing Times, 2005, 101 (1), 4

[18] Alive Technologies, Available online at www.alivetec.com, last accessed December, 2007

[19] Dongso Han, In-Youngko and Sungjoonpark, Information and Communications University, Daejon, Korea "An Evolving Mobile E-Health Service Platform" 
International Journal of Distributed and Parallel Systems (IJDPS) Vol.3, No.1, January 2012

[20] Evaluating the role of Primary Health Centers in India by Neesha Patel, Express Health care Management, 16-31 August 2005.

First Author: Karupothula Madhavi Latha is presently working as Asst.Prof in Computer Science Engineering Department at Jayamukhi Institute of Technological Sciences; Warangal for the past 5 years. She received her M.Tech Degree from JNTU Hyderabad,( A.P, India) in 2011.

Second Author: Shiramshetty Gouthami is presently working as Asst.Prof in Computer Science Engineering Department at Jayamukhi Institute of Technological Sciences; Warangal for the past 5 years. She received her M.Tech Degree from JNTU Hyderabad,( A.P, India) in 2011.

Third Author: Pogaku Ranjith Kumar is presently working as Asst.Prof in Computer Science Engineering Department at Jayamukhi Institute of Technological Sciences; Warangal for the past 5 years. He received his M.Tech Degree from JNTU Hyderabad,( A.P, India) in 2009. 